\title{
Self-induction of epileptic seizures by eye-closure. Spectral analysis of concomitant EEG
}

\author{
DG WASTELL.*AJ WILKINS.† CEDARBY \\ From the Department of Medical Computation. The Medical School, Manchester, the MRC Applied Psychology Unit, \\ Cambridge, $\uparrow$ Rumwell Hospital, Wickford, $\doteqdot U K$.
}

SUMMARY The spectral characteristics of the EEG were studied in the period immediately preceding slow eye-closures associated with the self-induction of epileptiform activity. Power levels were compared with similar spontaneous eye-closures not associated with paroxysmal activity, with eye closures performed to command, and with EEG preceding eye-blinks. A transient rise in EEG power at low frequencies 1-2 seconds before the slow eye-closure was found to be reliably associated with the subsequent occurrence of paroxsymal activity. This finding suggests that eye-closure may not be initial event in the sequence culminating in paroxsymal activity.

Self-induction of epileptic seizures by slow closure of the eyes has been the subject of two recent papers.' 2 including one in this Journal. Analysis of EEG activity has to date relied on visual descriptive methods. This short report supplements the existing work by using computer methods to quantify the spectral characteristics of the EEG surrounding the eye-closures. Since it has been argued that the slow eye-closure may play a leading causal role in self-induction (rather than forming part of the seizure pattern itself), we compared the EEG power spectrum prior to slow eye-closures. with that prior to other movements of the periocular musculature.

\section{Method}

Data was analysed from four patient volunteers from the Runwell Hospital EEG Department in whom the EEG revealed slow closures of the eyes similar to those described by Darby et al.2 Two of the patients were males aged 13 years, and two female. aged 14 and 26 . Three were taking anticonvulsant medication. All had a provisional diagnosis of primary generalised epilepsy.

EEG was recorded (time constant $=1.2 \mathrm{~s}$ ) from a bipolar montage on FM tape for off-line analysis on a PDP 11/60. Following pre-filtering, the EEG was digitised at $128 \mathrm{~Hz}$ and stored on disk-file. A FORTRAN programme permitted "paging" through the data to locate events of interest. whereupon a time-varying spectral analysis was performed as follows. The spectrum of epochs 1 second in width ( 128 points) was computed from a starting point 3 seconds before the

Address for reprint requests: Dr DG Wastell. Turner Dental Hospital. University of Manchester. Bridgeford Street. Manchester. M15 6FH UK

Received 30 April 1982 and in revised form 1 August 1982

Accepted 20 August 1982 significant event. and at 1 second intervals until 3 seconds after the event. Epochs were de-meaned and cosine tapered. as appropriate. Limitations in resources restricted the analysis to two channels of EEG: a frontal channel (FP2-F4) and a parieto-occipital derivation (P4-02). The following classes of event were distinguished: eye-closure leading to epileptiform paroxsymal activity (spike and slow wave-ECPA), eye-closure not associated with paroxsymal activity (ECNOPA), and eye-blinks (BLINK) occurring within 20 seconds either side of eye-closures. but also not directly associated with PA. EEG recordings typically ran for 45 minutes. On average for the four subjects. 12, 14 and 24 occurrences were found for the events ECPA. ECNOPA and BLINK respectively. Amplitude spectra from the parieto-occipital channel were calculated on each occasion, and were sub-averaged to yield the following broad frequency bands: $0-1 \mathrm{~Hz}$ (I). $2-3 \mathrm{~Hz}$ (II). $4-6 \mathrm{~Hz}$ (III) and 7-13 $\mathrm{Hz}$ (IV). Amplitudes were normalised across subjects using scaling factors calculated from resting EEG levels.

\section{Results}

Spectral estimates are notoriously unreliable. For each class of eye-closure, amplitude values from individual trials for corresponding time periods were therefore averaged. The figure shows group mean results for the following time periods: from 3 to 2 seconds before closure $(-3)$, from 2 to 1 seconds before $(-2)$, in the immediately preceding 1 second $(-1)$, and for the 3 second period immediately following (P). To establish the reproducibility of our results, a two way ANOVA was performed at each frequency, with type of eye-closure (A) and time period prior to EC (B) as the factors. No main effect of type of eye-closure was found for either $0-1 \mathrm{~Hz}(\mathrm{~F}=3 \cdot 6$, $\mathrm{df}=2,6, \mathrm{p}>0.05$ ) or the other frequency bands (all $F<1)$. ANOVA also showed no variations in EEG across 

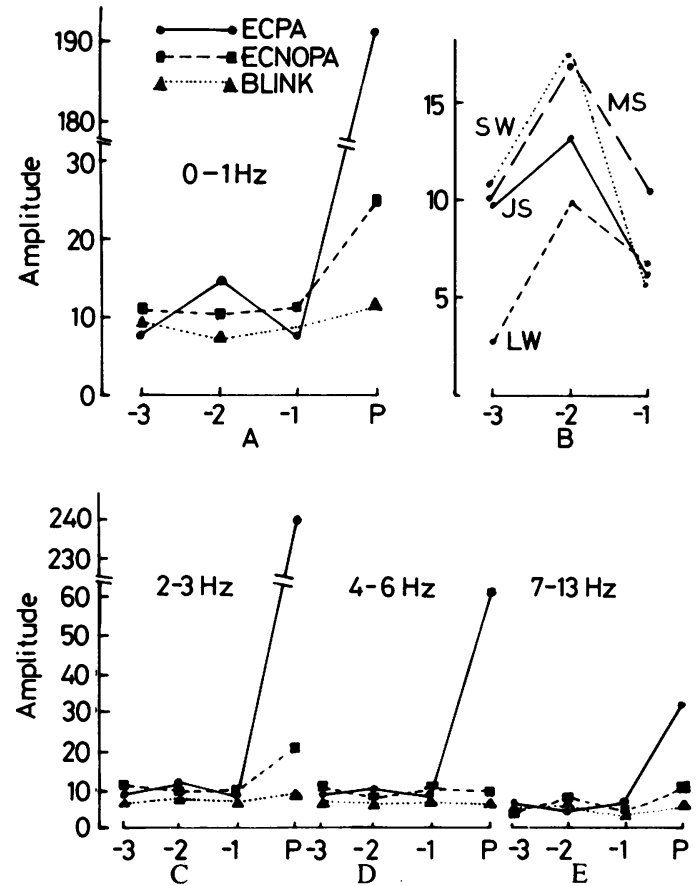

Figure (a)Amplitude (in arbitrary units after rescaling) of parieto-occipital EEG is shown for the 3 seconds immediately surrounding blinks (BLINK) and eye-closures with (ECPA) and without (ECNCPA) paroysmal activiry. The frequency bands $0-1,2-3,4-6,7-13 \mathrm{~Hz}$ are plotted separately in $A, C, D$ and $E$ respectively. 4 time regions are shown-the period from 3 to 2 seconds prior to eye-closure (denoted -3), 2 to 1 seconds before $(-2)$, in the immediately preceding 1 second $(-1)$, and for the 3 second period immediately following $(P)$. $(B)$ shows individual subjects data $(0-1 \mathrm{~Hz})$ for the 3 seconds prior to eve-closures resulting in $P A$.

the 3 second fore-period for all frequercy bands, with the main effect of time consistently failing to approach significance $(\mathrm{F}<1$ in all cases). For frequencies above 1 $\mathrm{Hz}$, no interaction between factors $\mathrm{A}$ and $\mathrm{B}$ was significant $(F<1.3$ throughout, $p>0 \cdot 25)$. However, for the $0-1 \mathrm{~Hz}$ band this interaction did prove reliable $(\mathrm{F}=$ $3 \cdot 7$, df $=4,12, p<0.05$ ), indicating that significant differences exist for the different types of eye-closure in the behaviour of low EEG frequencies in the period prior to closure. Further examination of the $\mathrm{A} \times \mathrm{B}$ interaction for the $0-1 \mathrm{~Hz}$ band revealed a quadratic trend in EEG amplitude for the ECPA condition (with EEG rising transiently 1-2 seconds before $E C$, and then falling away) but not for either ECNOPA or BLINK. Interaction contrast analysis ${ }^{34}$ showed this effect to be highly reliable $(\mathrm{F}=13 \cdot 2, \mathrm{df}=1,12, \mathrm{p}<0.01)$, accounting for $87 \%$ of the interaction variance. Figure (B) shows it to be consistently visible in the data of individual subjects. Inspection of the concomitant eye-field channel revealed no parallel effect, showing it to reflect brain rather than ocular activity.
Following eye-closure in the ECPA condition, the figure (ACDE) shows paroxysmal activity to be associated with a dramatic increase in EEG amplitude at all frequencies. Some increase in amplitude at the lower frequencies is also noted for ECNOPA (and to a much smaller extent for BLINKS in the $0-1 \mathrm{~Hz}$ band) suggesting the increase in power in ECPA at the lower end of the spectrum to be due not only to paroxysmal activity but also to volume-conducted activity from the eye-fields. This contribution is apparently small and restricted to $0-1$ $\mathrm{Hz}$.

Finally, EEG characteristics associated with eyeclosures performed to command were analysed from a sequence of such trials run separately from the main body of the recording. Although the electrical characteristics of these eye-closures differed from the slow eye-closures above, they provided further control data in that EEG power in their fore-period showed the same overall level as the other conditions, and there was no evidence of the distinctive quadratic trend of the ECPA data.

\section{Discussion}

In the four patients studied, a transient increase in low frequency EEG amplitude, occurring 1-2 seconds before slow eye-closure, was consistently and reliably observed on those closures that were followed by paroxysmal activity. This was not seen before other slow eye-closures or blinks, nor was it present in frequency bands other than $0-1 \mathrm{~Hz}$. Given the low frequency character of the phenomenon together with its temporal relationship to overt behaviour, we speculate that it may reflect the launching of a CNV-type d.c. potential shift ${ }^{5}$ such as are observed to accompany preparation for action. Appropriate CNV techniques will now be required to characterise more fully the phenomenon, but the present findings are at least sufficient to call into question the notion previously advanced that eye-closure is the first link in a causal chain that culminates in paroxysmal activity.

We thank Dr CD Binnie, Mr R Laxmenarayam and Dr AD Baddeley for their helpful comments on earlier versions of the paper.

\section{References}

' Binnie CD, Darby CE, de Korte RA, Wilkins AJ. Self induction of epileptic seizures by eyeclosure: incidence and recognition. J Neurol Neurosurg Psychiatry 1980;43:386-9.

${ }^{2}$ Darby CE, de Korte RA, Binnie CD, Wilkins AJ. The self induction of epileptic seizures by eye-closure. Epilepsia 1980;21:31-42.

${ }^{3}$ Boik RJ. Interactions, partial interactions, and interaction contrasts in the analysis of variance. Psychol Bull 1979:86: 1084-9.

${ }^{4}$ Wastell DG, Kleinman D. Potentiation of the habituation of human brain potentials. Biol Psychol 1980;10:21-9.

${ }^{5}$ Walter WG. Slow potential changes in the human brain associated with expectancy, decision and intension. Electroencephalogr Clin Neurophysiol 1967;Supple 26:123-30. 